\title{
A Trade-by-Trade Surprise Measure and Its Relation to Observed Spreads on the NYSE
}

\author{
Valeri Voev* \\ University of Konstanz, CoFE
}

14 March 2006

\begin{abstract}
We analyze the relationship between spreads and an indicator for information based transactions on trade-by-trade data. Classifying trades on the NYSE in six categories with respect to their volume relative to the quoted depth, we employ an ordered probit model to predict the category of a trade given the current market conditions. This approach allows us to test certain market microstructure hypothesis on the determinants of the buy-sell pressure. The difference between the predicted and the actual trade category (the surprise) is found to have explanatory power for the observed spreads beyond raw volume, volume relative to the quoted depth, and previous trading volume. The positive effect of the previous surprise on the observed spreads confirms the hypothesis that market-makers react to the increased probability of having traded with an informed trader by widening the spread.
\end{abstract}

\section{Introduction}

Since the availability of detailed order book data, the dynamics of the order flow and its interactions with the return process, the price volatility, and the presence of informed traders have been central issues concerning limit order book markets. Many studies have also been concerned with the order submission strategies of the traders given current market conditions as the bid-ask spread, or the order queues in the book 1 . The effect of informed traders on market liquidity and efficiency is controversial. On the one hand, informed traders make prices to reflect the information they have, which more often than not leads to prices which are closer to fundamental values. On the other hand liquidity traders and market-makers are more often than not losing to informed traders, which leads to larger bid-ask spreads and higher costs of trading. To empirically test whether information-based trading really influences observed variation in bid-ask spreads one needs a good measure for the presence of information. An

\footnotetext{
${ }^{*}$ Tel.: 0049753188 2556; E-mail: valeri.voev@uni-konstanz.de. Financial support from the Deutsche Forschungsgeimeinschaft, Research Group "Preis-, Liquiditäts- und Kreditrisiken: Messung und Verteilung" is gratefully acknowledged. I am thankful for comments to Winfried Pohlmeier and Jens Jackwerth. All remaining errors are mine.

${ }^{1}$ e.g. Harris and Hasbrouck (1996), Hall and Hautsch (2004), Bisière and Kamionka (2000)
} 
important property of such a measure is that it should reveal for each trade, whether it is likely to be information based. This is necessary, if one is to make conclusions about the influence of informed trades on subsequent behavior of market participants. Simple measures could be order arrival intensity, raw traded volume, or traded volume relative to quoted depth (see Heflin and Shaw (2004)). Easley, Kiefer, O'Hara, and Paperman (1996) developed a structural model in which the probability of information-based trading can be estimated from the order flow over a given time period. In their approach, an information event occurs with some probability and, if it occurs, it could be bad news or good news. The arrivals of informed and uniformed traders are assumed to be determined by Poisson processes with specific arrival rates. The model is easy to estimate and has shown that more infrequently traded stocks have a higher probability of informed trading. Furthermore it allows for an analysis of informed trading over time. One possible drawback, however, is that conditioning only on order flow can lead to some misleading results. To illustrate this point, assume that we have a booming economy. Under this condition there are more buys than sells and stock prices are systematically rising. Given such conditions a positive imbalance between buys and sells would be more attributable to public information about the macroeconomic conditions, rather than on particular positive news known only by a small portion of the traders. Consequently, a market maker would not be surprised to receive a buy order and would not have big incentives to alter the adverse selection fraction of the spread. Thus order imbalance is not necessarily a consequence of informed trading. In order to classify a trade as informed or not it is necessary to take into account not only the order imbalance, but also other characteristics describing the market conditions. In the previous example it would be appropriate to condition for example on the return of a broad economy index. Furthermore, the model uses aggregated data and does not deliver any insights as to whether a particular trade is information-based or not.

Many studies have addressed how trades influence the subsequent quote revision. Lee, Mucklow, and Ready (1993) recognize that both quoted prices and quoted depths should be viewed as important characteristics of market liquidity and the quote setting behavior of the dealers. They show that spreads increase and depths drop in response to an increase in volume. Hasbrouck (1991) argues that the informational content of a trade must be inferred not from total trade size but from the part which was unanticipated. He, however, excludes the relation of trade size to the quoted depth, which has some consequences on the quote revision process. This has been shown by Petersen and Umlauf (1994). They find that most of the variation in quote revision is explained by whether the trade size exceeds the quoted size. Furthermore, Kavajecz and Odders-White (2001) analyze what sources of information the specialist uses when updating the his quote prices and sizes. They also find that transactions that exceed the quoted depth contain more information and induce larger quote revisions.

Our contribution to this literature is to link the surprise or unanticipated component of trades to the quote revision process. Our framework allows for including the effects of the relation of quote size to trade size in classifying trades. Another important advantage of the specification is the possibility of including a broad set of variables (information set), which determine the expected trade category. For example, Hasbrouck (1991) models the quote midpoint and signed trade volume as a bivariate VAR. Thus, the trade innovation is the component of the trade which cannot be explained by previous trades and quote revisions. This, however, is only a small part of the information set of the market participants, which includes also other aspects of the market, as current price volatility, trading activity (not only 
measured by the quantity of past trades, but also the time between transactions), market index movements, etc.

To address these issues we develop an empirical framework which allows us to capture the degree of unexpectedness of a given trade, conditional on the current market conditions. Trades are classified in six categories according to their direction and size relative to the quoted depth. Conditional on current market characteristics, each trade is predicted to fall into one of these categories. The surprise is defined as the difference between the predicted and the true outcome. We presume that there is a relationship between this surprise measure and the reaction of the market-maker measured by the spread revision. If a market-maker aims and expects balanced trading but faces one sided orders, this would probably mean that his spreads are such that informed traders can profit. Therefore he will react to this deviation of the order flow from his expectations. In order to analyze whether this surprise measure has an impact on spreads, we use regression analysis where as explanatory variables we include also raw traded volume, traded volume relative to quoted depth, and an aggregate volume measure, which serves as an indicator for short-run market activity.

The results show that there is a positive relationship between the unexpectedness of a trade and the consequent bid-ask spread, which confirms the hypothesis that the market-maker reacts to surprise by widening the spread in order to protect himself from informed traders. Moreover, once we control for autocorrelation in spreads, the volume variables mentioned above have little additional explanatory power.

Our approach also allows us to test the significance of certain indicators describing the current market conditions for the determination of the buy-sell pressure. Thus, for example, we can use likelihood-based tests to check whether spread (as hypothesized in Hall and Hautsch (2004)), volatility, or market activity have an impact on the net buy-sell pressure.

The structure of the paper is as follows: Section 2 discusses the determinants of trade size relative to the quoted depth. Section 3 contains an overview of the data and discusses some problems arising from the use of a particular trade classification algorithm. In Section 4 we estimate a model for the information of informed trading in order to confirm some findings in the literature. Section 5 contains the estimation results. In the next section a measure of the degree of unexpectedness of a given trade is defined, which serves as a proxy for the probability of that trade being informed. Its relation to spreads is also analyzed. The last section concludes.

\section{Trade Size Relative to Quoted Depth and Its Determinants}

An underlying assumption of our model is that there is an agent, who bases his decision to trade on some information, available at the time of the decision. We will call this information set "the current market conditions". The set of market conditions include the current bid and ask prices, market activity, volatility, market movements and so on. Of course, this decision is driven also by liquidity needs and private information, which are unobservable for the marketmaker posting quotes. The market maker quotes in an optimal way (observing a given set of rules of the exchange), which means that he can influence the decision of the traders in order to maintain some optimal holdings of the stock and to protect himself from losses. If 
the direction and/or the size of next trade deviate from what he expected and aimed at, the market-maker can make some inference about the information content of the trade and possibly react by changing his quote. This change reflects the change in beliefs and deviation from some optimal holding of the stock.

In this paper, we define the types of trades according to their direction (buy or sell) and relation to the quoted depth. More precisely, letting the type of trade be denoted by $Z_{t}^{2}$ there are six possible values:

1. $Z_{t}=1-$ a sale of more shares than the current bid size,

2. $Z_{t}=2-$ a sale of the same number of shares as the current bid size,

3. $Z_{t}=3$ - a sale of less shares than the current bid size,

4. $Z_{t}=4-$ a buy of less shares than the current ask size,

5. $Z_{t}=5-$ a buy of the same number of shares as the current ask size,

6. $Z_{t}=6-$ a buy of more shares than the current ask size ${ }^{3}$.

In order to specify a meaningful econometric tool to model $Z_{t}$ we assume that there is an unobservable variable, whose outcome determines the outcome of $Z_{t}$. In our case this unobservable variable can be interpreted as the readiness of the agent to buy a given number of shares relative to the current bid size ${ }^{4}$. Increasing values of this variable correspond to the discrete variable taking on a higher value (e.g. a large negative value for the readiness to buy would result in a large sale, the variable $Z_{t}=1$, etc.). Thus, the categories $Z_{t}$ are ordered and a suitable econometric specification for $Z_{t}$ is the ordered probit model. Appendix A describes the model and its estimation by maximum likelihood.

Next, we turn to the description of the determinants of trade outcome. We start with a broad set of market conditions consisting of the following variables:

- $S E L L S I Z E_{t}$ - the number of shares the specialist is willing to buy at his best bid price (in thousands of shares),

- $B U Y S I Z E_{t}$ - the number of shares the specialist is willing to sell at his best ask price(in thousands of shares),

- PREVVOL $L_{t-i}$ for $i=1,2,3$ - the signed volumes of the last three trades (in thousands of shares),

- SPREADt - the prevailing bid-ask spread at the time of the transaction,

- $S U M D U R_{t}$ - the sum of the last three trade durations (in seconds),

\footnotetext{
${ }^{2}$ Here $t$ is an index for transaction time. Although the usual notation is $t_{i}$, here this would only clutter the notation.

${ }^{3}$ The terms bid and ask size refer to the quantities quoted by the market maker at the best bid and ask price respectively.

${ }^{4}$ Negative values are interpreted as the readiness to sell.
} 
- $V O L A_{t}$ - the sum of the squared midquote changes over the last three trades,

- PORTRET $T_{t}$ - the return of a capitalization-weighted portfolio of stocks over the last five minutes prior to the trade.

Considering the first two variables it is important to mention that on the NYSE open book policy has been introduced since January $24^{\text {th }} 2002$. This allows investors to see the entire limit order book, with all its quote levels. For 2001, which is the period we investigate, the traders could only see the best bid and ask with their corresponding sizes. Since those quotes are binding, an investor willing to trade up to the quoted size received speed of execution and a firm price. For trades bigger than the quoted size, which were submitted electronically without further conditions about the execution price, the trader received speed of execution but also an uncertain price for the volume above the best quoted size, which could be executed at multiple price levels. This has a twofold implication: first, the investors could not condition their decisions on prices and depths beyond the best quote; and second and more interesting - given that the execution price for the volume above the best quoted size was uncertain, such an order would definitely imply some hastiness on the part of the trader. Usually impatient traders are informed traders.

We further include the variable $S U M D U R_{t}$ to capture current market activity, $V O L A_{t}$ as an indicator of short-term volatility and PORTRET $T_{t}$ as an indicator of recent market movement ${ }^{5}$.

Given this set of market conditions, we could hypothesize on their possible impact on the trade category. Depth is one of the dimensions of liquidity. Hence a larger depth will ceteris paribus most probably lead to larger trade volumes. The question we are addressing here, however, is not the absolute impact, but the relative increase in trade size (as a proportion of the quoted depth) as the depth increases. If the relationship were linear, this relative impact would be zero. Convex relationship, implying positive relative impact, has the interpretation that the eagerness to trade increases more than proportionally with the quoted depth. We do not find any meaningful reason to support this possibility. A concave relationship might be explained by an asymmetric information argument: when a market-maker offers a large number of shares at a comparatively low ask price (and a relatively unattractive bid), this means that he is willing to sell, or put it differently he invites traders to buy from him. This can either be because he has moved away from some optimal portfolio and wants to unload some shares of a given stock, or because he fears that he has taken the losing side in a previous informed transaction (in this case he has bought possibly overvalued shares) and wants to reverse his position as soon as possible to curb his losses. This informational content of an asymmetric depth might hold an investor off from trading on the "attractive" side of the quote. Thus we expect either a negative sign or zero for the BUYSIZE variable, which can be interpreted as a test of linearity against concavity of the relationship between depth and trade size. Analogously we can argue about the positivity of SELLSIZE coefficient ${ }^{6}$.

The next plausible hypothesis is that positive past volumes (buy trades) have a positive impact on the buying pressure. This can be supported by the herding behavior (or momentum)

\footnotetext{
${ }^{5}$ Replacing this variable with the stock's own return does not change the results significantly.

${ }^{6} \mathrm{In}$ an ordered probit model the signs of the coefficients are not so straightforward to interpret. At least for the two extreme categories, however, a positive coefficient implies a smaller probability for the first and a larger probability for the last category. See Appendix A and Greene (1997).
} 
found in stock markets. Bikhchandani and Sharma (2000) discuss different reasons for this phenomenon, one of which is the informational explanation. Thus an investor might copy other investors if she believes that others have traded on some information. On the one hand, informed investors have an incentive to hide their trades among the liquidity trades. Thus, if they have observed a positive signal, they will prefer to buy when others are buying. This makes it hard for the market-maker to distinguish between informed and uninformed trading. On the other hand, value traders (see Harris (2003), Chapter 8 for a discussion of different types of informed traders) will trade if the price deviates from their estimate of fundamental values. Thus a sequence of buys which drives the price upwards, may cause value traders to sell if the price has risen substantially above their estimate. Thus trade reversals contain a higher probability of being informed.

A larger spread leads to lower liquidity and higher trading costs. Hence, it will lead to less trades both on the buy and the sell side. Hall and Hautsch (2004) find that spread is insignificant for explaining the buy-sell pressure. Although large bid-ask spreads make limit orders more attractive than market orders, there is no reason for buy- or sell-initiated orders to be affected in a different manner. We also argue that a larger spread most probably leads to smaller trades, but this impact is symmetric on both sides of the market, and hence no particular direction of the coefficient can be expected.

Market activity in terms of the frequency of trading, after having accounted for the direction and the volume of past trades, is also not expected to affect the buy and sell pressure in an asymmetric way. Higher volatility might make the stock unattractive to investors and hence induce less buying. Consequently, a negative coefficient is hypothesized.

The last variable in the conditional mean specification is the return of a capitalization weighted portfolio of the 24 stocks over the 5 minutes preceding the current trade. More precisely, we sample the trade prices at one minute intervals using last-tick interpolation. From the series of equally spaced 1-minute price observations we construct a series of 1minute log-returns. For each trade we find the price at the beginning of the minute the trade occurred, and sum the five preceding 1-minute log-returns. For example, if a trade occurred at 10:37:05 the variable PORTRET will be the log-return of the portfolio from 10:32:00 to 10:37:00. Economic intuition suggests that there will be more buys when the market is rising. This is the so called "momentum trading". Chan, Jegadeesh, and Lakonishok (1996) find several explanations for the profitability of momentum strategies. One of their findings is that markets absorb new information slowly. Especially, they note that a positive earnings announcement surprise is on average followed by surprises in the same direction at least over the next two subsequent announcements. There are, however, traders who employ contrarian strategies. That means they buy when the price is falling and sell when the price is rising. These traders have a stabilizing influence on prices. For this reasons it is hard to say a priori what the sign on the coefficient of PORTRET should be.

\section{The Data}

The data we use is extracted from the NYSE TAQ (Trades and Quotations) Database for the period January $2^{\text {nd }}$ to December $30^{\text {th }}$ of 2001, consisting of 248 trading days. The TAQ database consists of quotations and trade datasets for each stock traded on the NYSE, AMEX 
or the US regional exchanges. For our analysis we select the following 24 stocks with different levels of trading activity: Alcoa (NYSE ticker symbol: AA), Boeing (BA), Black \& Decker (BDK), Caterpillar (CAT), Citigroup (C), Colgate Palmolive (CL), Du Pont (DD), Walt Disney (DIS), Fair Isaac (FIC), General Electric (GE), Jack in the Box (JBX), J. P. Morgan Chase(JPM), Coca Cola (KO), Lockheed Martin (LMT), McDonalds (MCD), 3M (MMM), Altria Group (MO), Merck (MRK), Nike (NKE), Procter \& Gamble (PG), SBC Communications (SBC), AT\&T (T), United Tech (UTX), Wal-Mart Stores (WMT). The stocks were chosen at random, but in such a way that we have variation in their trading activity and traded volume.

The trade database contains the time, volume, and price of each trade. The quote database comprises the time of the quote update, the bid price, the ask price, and the corresponding volumes on the two sides. The sample precedes the introduction of the open book policy on the NYSE. Thus, the absence of information about the entire limit order book is not particularly harmful here. This is so, because at that time investors did not see the limit book either, hence this information could not be in their information set. This is illustrated in Figure 1. The bid and ask prices as well as the corresponding depths (represented by thick lines) were known to the public, while limit orders placed at worse prices (drawn in dashed lines) were not. The most severe drawback of this type of dataset for our purposes, is that it is not immediately clear whether the trade was buyer or seller initiated. This, combined with some delay of trade reporting can lead to problems with trade classification. To address this problem, we use the Lee and Ready (1991) algorithm which has been shown to perform reasonably well, although misspecifying a certain number of trades. It should be noted that here this problem is further aggravated by the fact that we also use the ratio of traded volume to the size of the corresponding quote to categorize the transactions in the six categories. This will inevitably lead to some trades falling into the wrong categories.

To make this point clearer, we illustrate it by the following example. Consider that the stock XYZ at 09:59:59 is quoted 99.90 (1000 shares) bid and 100.00 (500 shares) ask. Three seconds later, a limit sell order for 1000 shares at 100.00 is entered. Thus, the quotes at 10:00:02 are bid 99.90 (1000 shares) and ask 100 (1500 shares). Now, consider that a trade report is recorded at 10:00:05 with a price of 100.00 and a quantity of 1200 shares. According to the Lee and Ready (1991) algorithm this will be classified as a buyer initiated trade. In this case, concerning the classification of the trade, it is of no importance whether the valid quote at the time of the market order was the one at 09:59:59 or at 10:00:02. In any case this was a buy. For our purposes, however, judging by the rule that the quote which is at least 5 seconds old is the correct one, will lead us to categorize the trade as a buy larger than the size at the ask side (category 6). If, however, in this case the record was not actually delayed and the true quote was really the one at 10:00:02, then the trade should be classified as a buy smaller than the ask size (category 4). It is reasonable to assume that among the misspecified trades the ones that have been too extremely categorized (categories 1 and 6), will be more than the ones too moderately categorized.

These classification-induced errors will have as a consequence that the shares of the extreme categories will be more likely biased upwards at the expense of the middle ones. As a result this can only make the predictive performance of the ordered probit model worse than it actually is, since it turns out that the percent correctly predicted cases is the lowest for the extreme categories. Therefore, our results will most likely be understated, and would be more 


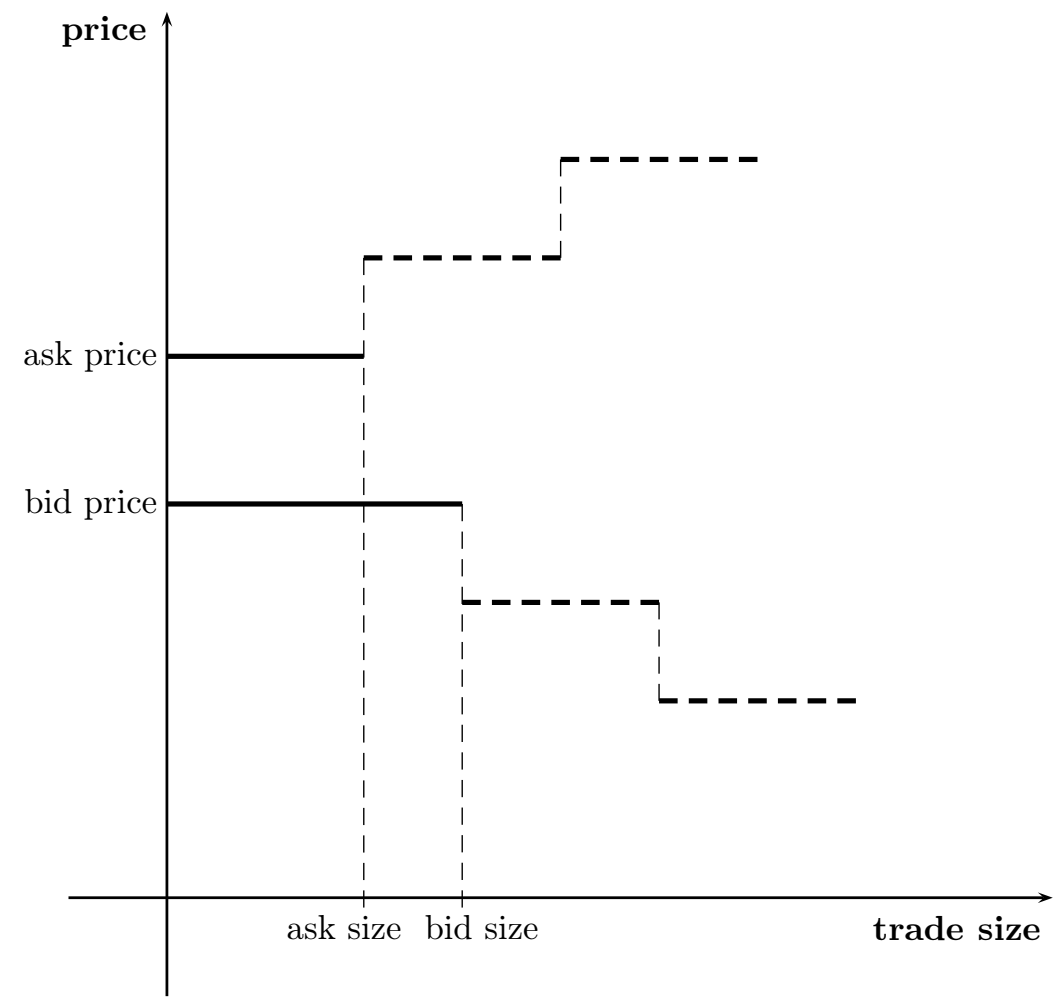

Figure 1: A snapshot of the limit order book at a given point of time.

Note: The figure shows a representation of an order book at a given point of time. Bid and ask price refer to the best prices at which an investor could sell or buy. Bid size and ask size are the depths, corresponding to the bid and ask prices, which were known to investors. The dashed lines represent limit orders with prices worse than the bid and ask price. These orders were not disclosed to the public. 
Table 1: Total number of trades and percentages of trades in the six categories.

\begin{tabular}{|l|r|r|r|r|r|r|r|r|}
\hline & AA & CAT & FIC & KO & MCD & MO & NKE & BDK \\
\hline \% trades in Cat. 1 & 0.18 & 0.14 & 0.13 & 0.20 & 0.15 & 0.16 & 0.14 & 0.11 \\
\% trades in Cat. 2 & 0.06 & 0.07 & 0.12 & 0.07 & 0.06 & 0.04 & 0.08 & 0.10 \\
$\%$ trades in Cat. 3 & 0.22 & 0.23 & 0.20 & 0.22 & 0.28 & 0.26 & 0.22 & 0.24 \\
\% trades in Cat. 4 & 0.30 & 0.32 & 0.28 & 0.24 & 0.31 & 0.33 & 0.31 & 0.34 \\
\% trades in Cat. 5 & 0.06 & 0.08 & 0.14 & 0.07 & 0.06 & 0.05 & 0.09 & 0.10 \\
\% trades in Cat. 6 & 0.18 & 0.16 & 0.14 & 0.20 & 0.15 & 0.17 & 0.16 & 0.12 \\
\hline Total number of trades & 361054 & 304764 & 62847 & 403178 & 427090 & 469120 & 211700 & 135673 \\
\hline \hline & CL & JBX & LMT & BA & C & DIS & GE & JPM \\
\hline \% trades in Cat. 1 & 0.14 & 0.14 & 0.14 & 0.16 & 0.17 & 0.17 & 0.19 & 0.19 \\
\% trades in Cat. 2 & 0.08 & 0.09 & 0.07 & 0.06 & 0.03 & 0.06 & 0.03 & 0.04 \\
\% trades in Cat. 3 & 0.23 & 0.21 & 0.23 & 0.23 & 0.26 & 0.26 & 0.25 & 0.21 \\
\% trades in Cat. 4 & 0.30 & 0.32 & 0.31 & 0.31 & 0.34 & 0.28 & 0.29 & 0.31 \\
\% trades in Cat. 5 & 0.09 & 0.10 & 0.08 & 0.06 & 0.03 & 0.06 & 0.03 & 0.05 \\
\% trades in Cat. 6 & 0.15 & 0.15 & 0.16 & 0.17 & 0.17 & 0.18 & 0.21 & 0.20 \\
\hline Total number of trades & 293132 & 52818 & 233623 & 418831 & 765039 & 483788 & 772361 & 627963 \\
\hline \hline & MMM & MRK & PG & T & WMT & UTX & SBC & DD \\
\hline \% trades in Cat. 1 & 0.17 & 0.23 & 0.16 & 0.14 & 0.19 & 0.18 & 0.17 & 0.15 \\
\% trades in Cat. 2 & 0.08 & 0.06 & 0.08 & 0.03 & 0.05 & 0.08 & 0.05 & 0.07 \\
\% trades in Cat. 3 & 0.19 & 0.19 & 0.25 & 0.36 & 0.21 & 0.19 & 0.27 & 0.26 \\
\% trades in Cat. 4 & 0.28 & 0.22 & 0.29 & 0.30 & 0.28 & 0.28 & 0.28 & 0.30 \\
\% trades in Cat. 5 & 0.09 & 0.06 & 0.08 & 0.03 & 0.06 & 0.08 & 0.05 & 0.07 \\
\% trades in Cat. 6 & 0.19 & 0.24 & 0.16 & 0.14 & 0.20 & 0.19 & 0.18 & 0.16 \\
\hline Total number of trades & 377775 & 478970 & 458499 & 453245 & 577617 & 326531 & 550948 & 449139 \\
\hline
\end{tabular}

pronounced if we could have an error-free trade classification. The distribution of the trades in the six categories described above is given in Table 1.

Due to the reasons discussed above we expect that due to the trade classification algorithm, some of the trades which should have been classified in categories 2 to 4 have migrated to the extreme categories 1 and 6 . Thus we expect that especially categories 2 and 5 are in fact larger than what is reported in the table.

\section{A Structural Model For the Probability of Information}

The Easley, Kiefer, O'Hara, and Paperman (1996) (hereafter EKOP) model is a structural model which estimates the probability of information, based on the difference between the buy and sell order flow. It assumes that with a certain probability $(\alpha)$ in the beginning of the trading period there is new information in the market, which is revealed only to some traders. This information, if it exists, can be a bad signal with probability $\delta$. Further, the arrival rates of informed and uninformed traders are modelled as independent Poisson processes, with intensity $\mu$ and $\epsilon$, respectively. The parameter vector $\theta=\{\alpha, \delta, \mu, \epsilon\}^{\prime}$ is then estimated by maximum likelihood and an estimate for the probability of informed trading is given by

$$
\widehat{P I}=\frac{\hat{\alpha} \hat{\mu}}{\hat{\alpha} \hat{\mu}+2 \hat{\epsilon}} .
$$




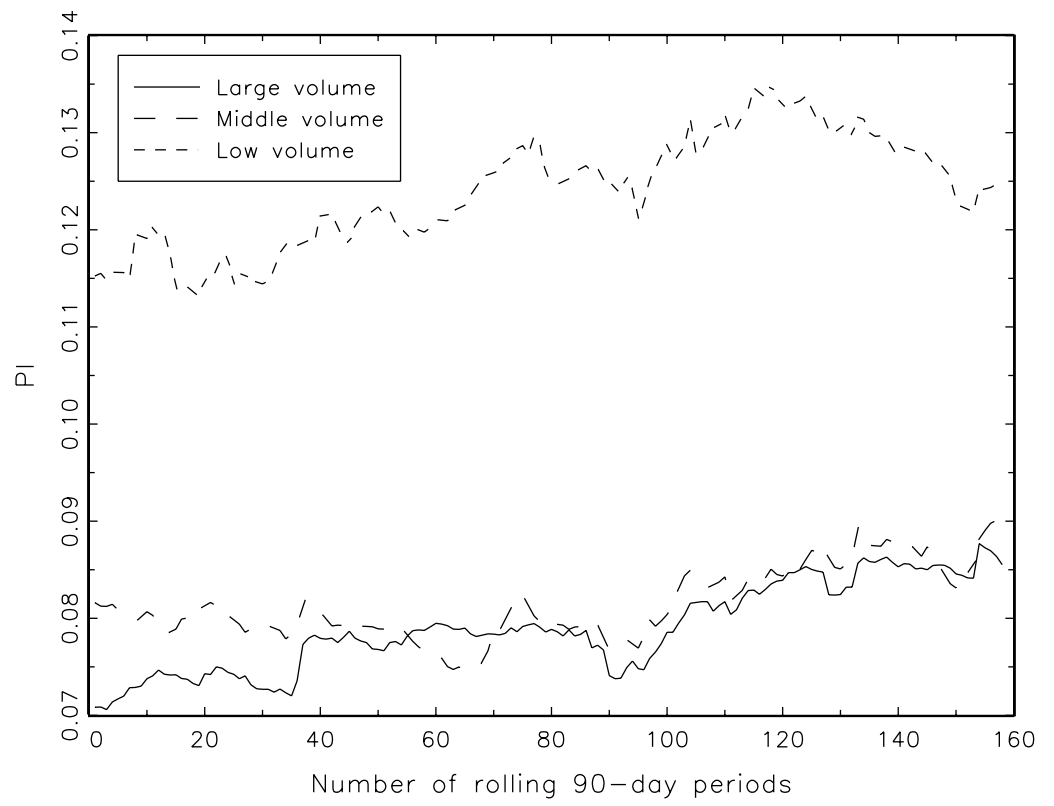

Figure 2: Probability of information for three volume quantiles.

Note: The figure plots the estimated probability of informed trading against 90-day rolling window estimation samples. The results are averaged over stocks in three volume quantiles.

We compute the number of buys and sells for each day in the period and estimate the model based on a rolling window of 90 days. Specific to the period in our study is that the number of buys was in general larger than the number of sells in almost all months. This led to the problem that in some cases the function reached a maximum when the parameter $\delta$ was on the lower boundary of its $[0,1]$ range. The booming US economy in that period led to heavy investment on the part of private and institutional investors. Thus the positive buy-sell order imbalance could hardly only be explained by positive private information, as found by the model. In general, our findings are similar to those in EKOP: grouping the stocks into three volume quantiles ${ }^{7}$, we find that the probability of news occurring is highest for the highest volume stocks, while the $\widehat{P I}$ is lowest. This is explained by the much larger intensity of arrival of uninformed traders in the large volume quantiles. Figure 2 plots the series of $\widehat{P I}$ as a function of the rolling 90-day windows. EKOP find in their sample of 90 stocks that stocks in the first volume decile have a significantly lower probability of informed trading compared to the fifth and eighth decile, while the difference between the fifth and eighth deciles is not significant. Since our sample of stocks is in general comprised of more active stocks than theirs, considering Figure 2 we also find support for the hypothesis that among the large stocks there is similar probability of informed trading. Both findings together indicate that it is likely that the stocks can be in general separated into two groups of similar probability - those with high probability (low volume stocks) and those with low probability (high volume stocks).

It is a little bit puzzling that our estimates of the probability of informed trading are far lower than what was found by EKOP. A possible explanation is that their sample is 11 years

\footnotetext{
${ }^{7}$ based on the overall traded volume
} 
Table 2: Average estimates, z-statistics and p-values for Alcoa

\begin{tabular}{|r|r|r|r|}
\hline Parameters & $\begin{array}{r}\text { Average } \\
\text { estimates }\end{array}$ & $\begin{array}{r}\text { Average } \\
z \text {-statistic }\end{array}$ & $\begin{array}{r}\text { Average } \\
p \text {-value }\end{array}$ \\
\hline SPREAD & -0.2681 & -1.0872 & 0.3008 \\
\hline SELLSIZE & 0.0378 & 20.5664 & 0.0000 \\
\hline BUYSIZE & -0.0291 & -21.2924 & 0.0000 \\
\hline PREVVOL1 & 0.0381 & 10.7702 & 0.0000 \\
\hline PREVVOL2 & 0.0114 & 6.4802 & 0.0003 \\
\hline PREVVOL3 & 0.0064 & 5.2802 & 0.0001 \\
\hline SUMDUR & -0.0001 & -0.5656 & 0.3178 \\
\hline VOLA & 0.0000 & 0.6826 & 0.2934 \\
\hline PORTRET & 42.9163 & 6.8116 & 0.0000 \\
\hline
\end{tabular}

Note: The table contains average coefficient values, $z$-statistics and $p$-values from 12 ordered probit estimations for Alcoa. The dependent variable is the trade category. Each estimation is based on a sample of one month. Estimates of the threshold parameters as well as a parameter of the conditional heteroscedasticity specification (see Appendix A) are not reported.

"older" than ours. It is a fact that within the period 1990-2000 the NYSE has succeeded in becoming a more transparent and active marketplace, which could be the reason for a decrease in informed trading as a proportion to overall trading activity. Furthermore, the NYSE has an active policy to prevent insider trading. Thus, these findings indicate that changes such as the decimalization since 19. January 2001 have enhanced market transparency and the price discovery process.

\section{Estimation Results and Interpretation of the Trade Category Model}

Initially we estimate the model based on monthly subsamples for each stock in order to confirm our expectations about the sign and significance of the variables based on large samples. The optimization converges for all stocks for almost all months. For the most heavily traded stock (General Electric) each monthly sample has approximately 55000 observations, while for the least active Fair Isaac (FIC) and Jack in the Box (JBX) there are some 3000 observations per month. The estimation results across stocks and subsamples are strikingly similar, which confirms the plausibility and robustness of the specification. From a total of approximately 250 estimation outputs we illustrate in Table 2 average coefficient values (from the 12 estimations based on monthly subsamples), as well as z-statistics and p-values for Alcoa.

The first result which is observed is that even though the samples are large, quite often the coefficients on SPREAD and SUMDUR are statistically insignificant even at $5 \%$ confidence levels, based on the $z$-statistic which is asymptotically standard normal under the null hypothesis, that the coefficient is equal to zero. These findings are consistent with our hypotheses in Section 2. The insignificance of the variable measuring current stock volatility, however, contradicts the intuition that high volatility will increase the sell pressure. Thus, although 
Table 3: Likelihood ratio test statistics and $p$-values for the joint significance of SPREAD, SUMDUR and VOLA for five stocks.

\begin{tabular}{|c|c|c|c|c|c|c|c|c|c|c|}
\hline & \multicolumn{2}{|c|}{ Alcoa } & \multicolumn{2}{|c|}{ Caterpillar } & \multicolumn{2}{|c|}{ Fair Isaac } & \multicolumn{2}{|c|}{ Coca Cola } & \multicolumn{2}{|c|}{ McDonald's } \\
\hline & LR & $p$-value & $\mathrm{LR}$ & $p$-value & LR & $p$-value & LR & $p$-value & LR & $p$-value \\
\hline January & 12.5363 & 0.0058 & 6.6390 & 0.0843 & 13.6570 & 0.0034 & 2.0790 & 0.5562 & 4.8624 & 0.1822 \\
\hline February & 2.4135 & 0.4911 & 4.3295 & 0.2280 & 2.7726 & 0.4280 & 6.5567 & 0.0875 & 0.6052 & 0.8952 \\
\hline April & 7.5889 & 0.0553 & 4.3602 & 0.2251 & 8.3672 & 0.0390 & 12.1519 & 0.0069 & 12.4978 & 0.0059 \\
\hline May & 13.4475 & 0.0038 & 38.8512 & 0.0000 & 3.2906 & 0.3490 & 5.8858 & 0.1173 & 23.5160 & 0.0000 \\
\hline August & 8.1531 & 0.0430 & 8.0236 & 0.0455 & 5.6235 & 0.1314 & 31.4016 & 0.0000 & 5.9818 & 0.1125 \\
\hline September & 5.9215 & 0.1155 & 19.8733 & 0.0002 & 2.6902 & 0.4419 & 11.1995 & 0.0107 & 7.3892 & 0.0605 \\
\hline October & 4.4314 & 0.2185 & 5.6451 & 0.1302 & 5.3961 & 0.1450 & 2.6964 & 0.4408 & 15.7912 & 0.0013 \\
\hline November & 3.3559 & 0.3399 & 16.1013 & 0.0011 & 5.2529 & 0.1542 & 15.2319 & 0.0016 & 21.1990 & 0.0001 \\
\hline December & 22.9867 & 0.0000 & 42.2005 & 0.0000 & 0.4827 & 0.9227 & 13.2989 & 0.0040 & 26.0638 & 0.0000 \\
\hline
\end{tabular}

high volatility might hold investors off from trading, it does not do so in an asymmetric way. To check the joint significance of the three parameters we conduct likelihood-ratio tests. Since the results look quite similar across stocks, we report the values of the test statistic as well as the corresponding $p$-value ${ }^{8}$ for five randomly chosen stocks in Table 3 . Considering the large samples, the table presents an overwhelming support for the conjecture that the three variables do not influence the buy and sell order submission asymmetrically. Of course, for stocks with fewer observations these conclusions are most obvious.

The coefficients on the depth variables are highly significant and have the signs we expected. This confirms the concave relationship between trade size and quoted depth. Thus we find support for the hypothesis that the market-maker's depth quotes contain information about his valuation of the stock or inventory imbalances.

The past volume variables also have the signs we expected. Positive past volumes (buy trades) have a positive impact on the buying pressure. This impact, however, quickly diminishes as we can see from the relative sizes of the coefficients ${ }^{9}$. This finding supports the herding behavior (or momentum) found in stock markets.

Further, we find evidence from the estimation results that the return of a stock portfolio has a significant positive impact on the buy-sell pressure. This is in line with the hypothesis that traders are more willing to buy when the market is going upwards. Thus, although there might be trading by contrarian traders, it is not enough to reverse the momentum caused by traders who buy when prices are rising and sell otherwise. Another important implication of the significance of this coefficient is that judging about the presence of private information solely by the trade imbalance, misses to account for an important factor driving this imbalance.

\footnotetext{
${ }^{8}$ The test statistic has a $\chi^{2}$ distribution with 3 degrees of freedom.

${ }^{9} \mathrm{We}$ can compare the sizes of these three coefficients, because the associated variables have the same measurement units - size of trade in thousands of shares.
} 


\section{A Trade-by-Trade Surprise Measure and Its Relation to Spreads}

The drawback of estimating the model based on monthly subsamples is that it implicitly assumes that the economic relation stays stable within such a long period. Fortunately, the great number of observations enables us to perform the model estimation on daily subsamples ${ }^{10}$. By considering smaller subsamples we add flexibility to the specification by allowing for temporal changes of the relationship. Examining the time series of coefficients it is clear that although they are quite stable, there is still some variation in time.

The ordered probit model can deliver predictions of the discrete variable $Z_{t}$ (the trade category), given the characteristics of a given trade. McKelvey and Zavoina (1975) suggest the following methodology to use the maximum likelihood estimates to predict the ordinal variable $Z_{t}$ : given a particular observation $X_{t}=\left(X_{1 t}, \ldots, X_{q t}\right)^{\prime}$, let $\hat{P}_{t, k}$ be the predicted probability that the dependent variable $Z_{t}$ is in the $k$-th category. This probability is computed as ${ }^{11}$ :

$$
\hat{P}_{t, k}=\Phi\left(\frac{\hat{\mu}_{k}-X_{t}^{\prime} \hat{\beta}}{\hat{\sigma}_{t}}\right)-\Phi\left(\frac{\hat{\mu}_{k-1}-X_{t}^{\prime} \hat{\beta}}{\hat{\sigma}_{t}}\right),
$$

where $\hat{\sigma}_{t}=\sqrt{1+\hat{\gamma}_{1}^{2} D_{t}}$. Letting $\hat{Z}_{t}$ be the maximum likelihood prediction of $Z_{t}$, we have

$$
\hat{Z}_{t}=k_{t},
$$

where $k_{t}$ is the value $1 \leq k_{t} \leq M$ which maximizes $\hat{P}_{t, k_{t}}$. A typical goodness-of-fit measure of ordered probit models is the percentage of correctly classified $Z_{t}$. A typical figure in our estimations is $40 \%$ with the percentage for categories 3 and 4 as high as $80 \%$, while for the extreme categories it drops down to several percent. This poor performance in predicting the extreme categories can be partially attributed to errors due to the trade classification algorithm. Of course, it is also to be expected that those trades contain the biggest proportion of surprise and are therefore hard to predict.

Having a prediction about the category of a trade, conditional on the prevailing market conditions at this point of time, we define a surprise measure as

$$
S_{t}=\hat{Z}_{t}-Z_{t} \text {. }
$$

Thus, a correctly predicted trade will have a zero surprise, while in the extreme case where a large buy is predicted but a large sell actually occurred, the surprise will take on a value of 5. This measure has a nice intuitive interpretation. If the market maker expects a buy, given his spread and depth and the market conditions, and he is confronted with a market sell order, then this unexpected event carries information and will most likely be reflected in the quote setting behavior of the market maker. Consequently, we expect a positive relationship between the absolute value of the surprise measure and the following spread ${ }^{12}$.

In order to check our hypothesis we run OLS regressions for each stock and each day, where we regress the spread of the $t^{t h}$ transaction on a constant, the previous surprise, and

\footnotetext{
${ }^{10}$ For the least active stocks we use two days of trading as a subsample.

${ }^{11}$ See Appendix A for details.

${ }^{12}$ As mentioned by Lee, Mucklow, and Ready (1993) on the NYSE the specialist's quote reflects the aggregate supply of liquidity from limit orders and standing orders, as well as his own trading intentions. Thus, his behavior represents that of all liquidity suppliers.
} 
Table 4: Number of significantly positive parameters in the OLS regression (1) under the restriction $\beta_{3}=\beta_{5}=0$ based on t-tests at $1 \%$ and $5 \%$ significance levels, respectively.

\begin{tabular}{|c|c|c|c|c|c|c|c|c|c|c|}
\hline & \multicolumn{2}{|c|}{ Walt Disney } & \multicolumn{2}{|c|}{ Citigroup } & \multicolumn{2}{|c|}{ GE } & \multicolumn{2}{|c|}{ Caterpillar } & \multicolumn{2}{|c|}{ Alcoa } \\
\hline & $1 \%$ & $5 \%$ & $1 \%$ & $5 \%$ & $1 \%$ & $5 \%$ & $1 \%$ & $5 \%$ & $1 \%$ & $5 \%$ \\
\hline$\beta_{1}$ & 147 & 174 & 234 & 239 & 221 & 229 & 58 & 92 & 62 & 92 \\
\hline$\beta_{2}$ & 139 & 161 & 108 & 119 & 112 & 133 & 134 & 163 & 172 & 192 \\
\hline$\beta_{4}$ & 123 & 137 & 221 & 230 & 178 & 187 & 75 & 90 & 109 & 127 \\
\hline & \multicolumn{2}{|c|}{ Boeing } & \multicolumn{2}{|c|}{ Coca Cola } & \multicolumn{2}{|c|}{ McDonald's } & \multicolumn{2}{|c|}{ Jack in the Box } & \multicolumn{2}{|c|}{ Lockheed Martin } \\
\hline & $1 \%$ & $5 \%$ & $1 \%$ & $5 \%$ & $1 \%$ & $5 \%$ & $1 \%$ & $5 \%$ & $1 \%$ & $5 \%$ \\
\hline$\beta_{1}$ & 145 & 179 & 73 & 103 & 91 & 126 & 16 & 29 & 24 & 38 \\
\hline$\beta_{2}$ & 104 & 128 & 169 & 188 & 141 & 172 & 17 & 30 & 46 & 65 \\
\hline$\beta_{4}$ & 115 & 128 & 105 & 134 & 78 & 94 & 28 & 39 & 43 & 51 \\
\hline
\end{tabular}

Note: The regression $S P R_{t}=\beta_{0}+\beta_{1} R A W S_{t-1}+\beta_{2} R E L S_{t-1}+\beta_{3}\left|S_{t-1}\right|+\beta_{4} V O L_{t-1}+\beta_{5} S P R_{t-1}+\varepsilon$, is estimated for each of the 248 days in the sample, except those for which we did not have estimates of the ordered probit model and $\left|S_{t-1}\right|$ was not available. The stock with the most such cases is GE with 7 days for which the maximum likelihood did not converge. For Jack in the Box and Lockheed Martin we have 120 regressions on 2-day samples.

several control variables. In particular, we consider the following linear regression

$$
S P R_{t}=\beta_{0}+\beta_{1} R A W S_{t-1}+\beta_{2} R E L S_{t-1}+\beta_{3}\left|S_{t-1}\right|+\beta_{4} V O L_{t-1}+\beta_{5} S P R_{t-1}+\varepsilon,
$$

where $S P R_{t}$ is the bid-ask spread at the time of the $t^{t h}$ transaction, $R A W S_{t}$ is the raw volume size (in thousands of shares), $R E L S_{t}$ is the trade volume relative to the quoted depth on the corresponding side of the market ${ }^{13}, S_{t}$ is our surprise measure, and $V O L_{t}$ is the accumulated volume of the last 100 trades (including the $t^{\text {th }}$ ). This variable was included since Lee, Mucklow, and Ready (1993) present some evidence that there is a positive relationship between past trading volume and spreads. The lagged spread is included, since we find strong positive autocorrelation in the spread series. We also tried adding more lags, but they are in most cases insignificant, so we find this specification to be the best in terms of parsimony and uncorrelatedness of the OLS residuals.

Given the specification in Equation 1, we expect positive signs for all coefficients. Heflin and Shaw (2004) find that raw trade size and high-trading-volume half-hours offer almost no explanatory power for informed trading measures beyond that of trade size to quoted depth. Therefore it is interesting to obtain results for the regression under the restriction $\beta_{3}=0$ and $\beta_{5}=0$. It turns out that the results and the conclusions vary with the stock trading activity. Therefore we report results for some stocks, which we classify again in three volume quantiles. Representatives of the low volume quantile are the stocks of Jack in the Box and Lockheed Martin, of the middle quantile - Alcoa, Boeing, Coca Cola, Caterpillar and McDonald's, and of the large volume quantile - Walt Disney, Citigroup and General Electric. Table 4 lists the number of significantly positive coefficients out of the $248^{14}$ regressions for each stock.

\footnotetext{
${ }^{13}$ For example, if there was a buy of 500 shares, while the depth at the ask side was 1000 shares, then $R E L S_{t}=0.5$.

${ }^{14}$ See table note for details.
} 
Table 5: Number of significantly positive parameters in the OLS regression (1) under the restriction $\beta_{3}=0$ based on t-tests at $1 \%$ and $5 \%$ significance levels, respectively.

\begin{tabular}{|c|c|c|c|c|c|c|c|c|c|c|}
\hline & \multicolumn{2}{|c|}{ Walt Disney } & \multicolumn{2}{|c|}{ Citigroup } & \multicolumn{2}{|c|}{ GE } & \multicolumn{2}{|c|}{ Caterpillar } & \multicolumn{2}{|c|}{ Alcoa } \\
\hline & $1 \%$ & $5 \%$ & $1 \%$ & $5 \%$ & $1 \%$ & $5 \%$ & $1 \%$ & $5 \%$ & $1 \%$ & $5 \%$ \\
\hline$\beta_{1}$ & 132 & 181 & 234 & 237 & 180 & 205 & 45 & 89 & 65 & 118 \\
\hline$\beta_{2}$ & 42 & 79 & 58 & 72 & 39 & 55 & 33 & 54 & 42 & 68 \\
\hline$\beta_{4}$ & 29 & 52 & 116 & 155 & 69 & 96 & 13 & 35 & 22 & 44 \\
\hline \multirow[t]{3}{*}{$\beta_{5}$} & 245 & 245 & 246 & 246 & 241 & 241 & 248 & 248 & 246 & 246 \\
\hline & \multicolumn{2}{|c|}{ Boeing } & \multicolumn{2}{|c|}{ Coca Cola } & \multicolumn{2}{|c|}{ McDonald's } & \multicolumn{2}{|c|}{ Jack in the Box } & \multicolumn{2}{|c|}{ Lockheed Martin } \\
\hline & $1 \%$ & $5 \%$ & $1 \%$ & $5 \%$ & $1 \%$ & $5 \%$ & $1 \%$ & $5 \%$ & $1 \%$ & $5 \%$ \\
\hline$\beta_{1}$ & 100 & 146 & 84 & 137 & 95 & 151 & 16 & 28 & 25 & 50 \\
\hline$\beta_{2}$ & 45 & 74 & 27 & 53 & 28 & 51 & 5 & 14 & 11 & 19 \\
\hline$\beta_{4}$ & 17 & 42 & 20 & 53 & 19 & 34 & 7 & 12 & 10 & 19 \\
\hline$\beta_{5}$ & 247 & 247 & 248 & 248 & 246 & 246 & 118 & 119 & 120 & 120 \\
\hline
\end{tabular}

Note: The regression $S P R_{t}=\beta_{0}+\beta_{1} R A W S_{t-1}+\beta_{2} R E L S_{t-1}+\beta_{3}\left|S_{t-1}\right|+\beta_{4} V O L_{t-1}+\beta_{5} S P R_{t-1}+\varepsilon$, is estimated for each of the 248 days in the sample, except those for which we did not have estimates of the ordered probit model and $\left|S_{t-1}\right|$ was not available. The stock with the most such cases is GE with 7 days for which the maximum likelihood did not converge. For Jack in the Box and Lockheed Martin we have 120 regressions on 2-day samples.

In order to check whether traded size relative to depth subsumes the information of raw trade size with respect to explaining the spreads, we need to compare the significance of the coefficients $\beta_{1}$ and $\beta_{2}$. If $\beta_{2}$ is always significant, while $\beta_{1}$ is not, then we find strong support for the hypothesis. From the table it can be seen that for the less active stocks, we rarely find significant coefficients (except the estimate for the mean). In this case, this is most likely a consequence of the small number of observations (on average 325 for Jack in the Box and 1780 for Lockheed Martin), which leads to large standard errors for the parameters. Still, we find that the $\beta_{2}$ was significantly positive in most cases than $\beta_{1}$. Turning to the middlevolume quantile, with the exception of Boeing, we find strong support that previous relative trade size explains better the next spread than the raw trade size. For the big companies, however, the results are just the opposite. One possible explanation is that for these stocks, the probability of informed trading is generally lower, markets are deeper, and thus the size of the market order seems to carry information which is by itself more relevant than its relation to the current depth.

Thus, without accounting for the serial properties of the spread, we find some support for the findings of Heflin and Shaw (2004). Relaxing the constraint $\beta_{5}=0$, however, leads to quite different conclusions. In Table 5 we perform the same analysis, this time with the single restriction $\beta_{3}=0$. The first result we observe is that for most of the stocks, both trade size measures become less significant once the autocorrelation is taken into account. This holds even stronger for the measure of the previous trading activity. Secondly, now we see that for all stocks, raw size is significant more often than relative size. In general, we can conclude that with the exception of the large-volume stocks, the three volume measures offer little additional explanatory power when the serial properties of the dependent variable are properly accounted for. 
Table 6: Number of significantly positive parameters in the OLS regression (1) based on t-tests at $1 \%$ and $5 \%$ significance levels, respectively.

\begin{tabular}{|r|r|r|r|r|r|r|r|r|r|r|}
\hline & \multicolumn{2}{|c|}{ Walt Disney } & \multicolumn{2}{|c|}{ Citigroup } & \multicolumn{2}{|c|}{ GE } & \multicolumn{2}{|c|}{ Caterpillar } & \multicolumn{2}{|c|}{ Alcoa } \\
\hline & $1 \%$ & $5 \%$ & $1 \%$ & $5 \%$ & $1 \%$ & $5 \%$ & $1 \%$ & $5 \%$ & $1 \%$ & $5 \%$ \\
\hline$\beta_{1}$ & 94 & 139 & 215 & 230 & 158 & 190 & 32 & 69 & 37 & 78 \\
$\beta_{2}$ & 27 & 64 & 43 & 62 & 34 & 47 & 22 & 42 & 40 & 64 \\
$\beta_{3}$ & 220 & 235 & 236 & 242 & 194 & 212 & 157 & 197 & 137 & 170 \\
$\beta_{4}$ & 26 & 50 & 120 & 160 & 64 & 96 & 12 & 32 & 18 & 40 \\
$\beta_{5}$ & 245 & 245 & 246 & 246 & 241 & 241 & 248 & 248 & 246 & 246 \\
\hline \hline & \multicolumn{2}{|c|}{ Boeing } & \multicolumn{2}{|c|}{ Coca Cola } & \multicolumn{2}{|c|}{ McDonald's } & Jack in the Box & Lockheed Martin \\
\hline & $1 \%$ & $5 \%$ & $1 \%$ & $5 \%$ & $1 \%$ & $5 \%$ & $1 \%$ & $5 \%$ & $1 \%$ & $5 \%$ \\
\hline$\beta_{1}$ & 83 & 115 & 67 & 112 & 52 & 92 & 16 & 23 & 17 & 34 \\
$\beta_{2}$ & 33 & 60 & 20 & 51 & 14 & 38 & 4 & 10 & 8 & 14 \\
$\beta_{3}$ & 178 & 212 & 128 & 162 & 231 & 241 & 18 & 41 & 101 & 113 \\
$\beta_{4}$ & 18 & 38 & 22 & 51 & 14 & 31 & 7 & 13 & 10 & 19 \\
$\beta_{5}$ & 247 & 247 & 248 & 248 & 246 & 246 & 118 & 119 & 120 & 120 \\
\hline
\end{tabular}

Note: The regression $S P R_{t}=\beta_{0}+\beta_{1} R A W S_{t-1}+\beta_{2} R E L S_{t-1}+\beta_{3}\left|S_{t-1}\right|+\beta_{4} V O L_{t-1}+\beta_{5} S P R_{t-1}+\varepsilon$, is estimated for each of the 248 days in the sample, except those for which we did not have estimates of the ordered probit model and $\left|S_{t-1}\right|$ was not available. The stock with the most such cases is GE with 7 days for which the maximum likelihood did not converge. For Jack in the Box and Lockheed Martin we have 120 regressions on 2-day samples.

Next, we consider the explanatory power of the surprise measure on spreads. Similarly, in Table 6 we list the number of positively significant coefficients for the unrestricted model. The results clearly indicate that the surprise measure which we construct, offers additional explanatory power and is by far the most important determinant of the next spread, after conditioning on the previous spread. With the exception of the smallest stock (Jack in the Box) in our whole sample of 24 stocks, we find strong support for the hypothesis that the specialist reacts to surprising trades by widening the spread. Of course, what we define as a "surprising trade" is not necessarily invoked by information, but the results clearly indicate that they have an impact on liquidity, making the market less tight. This has as a consequence larger trading costs for subsequent traders, since the market-maker will try to transfer the possible losses of trading with an informed trader to other market participants who trade afterwards.

\section{Conclusion}

This paper studies the relationship between a proxy for information based trading and spreads. The availability of trade and quote datasets allows for classifying the trades according to their relation to the quoted depth at the corresponding side of the market. Using a model for the analysis of ordinal level dependent variables, we specify a model for the conditional mean of trade categories, which is a function of current market conditions. These variables enter the information set of the investor and determine his trading behavior. The dataset limitations notwithstanding, we find that the model performs well in terms of predicting the middle categories. Furthermore, the likelihood based estimation technique allows for tests of market 
microstructure hypotheses about the impact of certain market variables as volatility, and market activity on the buy-sell pressure. Our results indicate that the prevailing spread at the time of the transaction does not affect the buy and sell pressure asymmetrically, which was also found in other empirical studies. Furthermore, we find support for the hypothesis that short term market volatility and trading intensity do not influence the net buy-sell activity.

The central problem considered in the study is the reaction of spreads to surprising trades. A surprise measure is constructed by taking the difference between the prediction of the next trade category, based on the estimates of an ordered probit model and the category of the actual trade. Large absolute values of this measure indicate that the market maker (or a standing limit order) needs to take the other side of an incoming market order, which was unlikely to be submitted given the current market conditions. Unless having additional information about who and for what reasons would be willing to trade at the "wrong" time, the specialist attaches to such an order a greater probability of stemming from an informed trader. Subsequently, he either quotes a larger spread, or nobody is willing to submit an aggressive limit order, which leaves the spread wider. This is so, because if the trade was really initiated by an informed trader, offering a better price again would lead to that trader trading again and the other side losing.

Existing models for the probability of informed trading rely mostly on aggregated trade data, which render them unsuitable to infer this probability on the trade-by-trade level. Although we do not quantify this probability, with our approach we can classify single trades as ones with relatively low or high probability of being informed, depending on the degree of surprise.

Regression results indicate that our surprise measure is the most important determinant of spreads, once their serial properties have been accounted for. In particular, it can be shown that previous trading activity as well as raw and relative trade size deliver little additional explanatory power, except for large-volume stocks. 


\section{A Econometric Model}

The ordered probit model was introduced by McKelvey and Zavoina (1975) to analyze ordered discrete dependent variables. The model was later applied in the field of market microstructure by Hausman, Lo, and MacKinlay (1992) and Pohlmeier and Gerhard (2000), among others, to analyze the determinants of transaction stock prices and volatility, and by Beber and Caglio (2003) who investigate order submission strategies based on current market conditions.

\section{A.1 Description of the Model}

Let $Z_{t}^{*}$ be a continuous random variable. The conditional mean of $Z_{t}^{*}$ is driven by some predetermined variables $X_{t}$, a $q \times 1$ vector:

$$
Z_{t}^{*}=X_{t}^{\prime} \beta+\varepsilon_{t}, \quad \mathrm{E}\left[\varepsilon_{t} \mid X_{t}\right]=0, \quad \varepsilon_{t} \quad I N I D \quad N\left(0, \sigma_{t}^{2}\right) .
$$

Instead of observing $Z_{t}^{*}$, we observe the discrete variable $Z_{t}$, which is related to its continuous counterpart in the following manner:

$$
Z_{t}=\left\{\begin{array}{lll}
s_{1} & \text { if } & Z_{t}^{*} \in\left(-\infty, \mu_{1}\right] \\
\vdots & & \vdots \\
s_{i} & \text { if } & Z_{t}^{*} \in\left(\mu_{i-1}, \mu_{i}\right] \\
\vdots & & \vdots \\
s_{M} & \text { if } & Z_{t}^{*} \in\left(\mu_{M-1}, \infty\right)
\end{array}\right.
$$

where $\mu_{i}, \quad i=0, \ldots, M$ are threshold parameters with $\mu_{0}=-\infty$ and $\mu_{M}=\infty$.

As noted in Equation (2) we let the error term be conditionally heteroscedastic. This is mainly motivated by the irregularly spaced observations which led us to assume a duration dependent conditional variance of $\varepsilon_{t}$, given by $\sigma_{t}=\sqrt{\gamma_{0}^{2}+\gamma_{1}^{2} D_{t}}$, where $\gamma_{0}=1$ for identification purposes (see Hausman, Lo, and MacKinlay $(1992)^{15}$ ) and $D_{t}$ is the duration in seconds between the trade at time $t$ and the previous one.

Given this structure of the model, the distribution of $Z_{t}$, assuming normally distributed

\footnotetext{
${ }^{15}$ This has to do with the fact that the likelihood is unchanged if the threshold parameters, the $\beta$ 's and $\sigma_{t}$ are multiplied by the same number. This implies that the parameters are estimated up to a scaling factor $1 / \gamma_{0}$.
} 
$\varepsilon_{t}$ 's, is:

$$
\begin{aligned}
\operatorname{Pr}\left[Z_{t}=s_{i} \mid X_{t}, D_{t}\right]= & \operatorname{Pr}\left[X_{t}^{\prime} \beta+\varepsilon_{t} \in A_{i} \mid X_{t}, D_{t}\right] \\
= & \begin{cases}\operatorname{Pr}\left[X_{t}^{\prime} \beta+\varepsilon_{t} \leq \mu_{1} \mid X_{t}, D_{t}\right] & \text { if } \quad i=1 \\
\operatorname{Pr}\left[\mu_{i-1}<X_{t}^{\prime} \beta+\varepsilon_{t} \leq \mu_{i} \mid X_{t}, D_{t}\right] & \text { if } \quad 1<i<M \\
\operatorname{Pr}\left[\mu_{M-1}<X_{t}^{\prime} \beta+\varepsilon_{t} \mid X_{t}, D_{t}\right] & \text { if } \quad i=M\end{cases} \\
= & \begin{cases}\Phi\left(\frac{\mu_{1}-X_{t}^{\prime} \beta}{\sigma_{t}}\right) & \text { if } \quad i=1 \\
\Phi\left(\frac{\mu_{i}-X_{t}^{\prime} \beta}{\sigma_{t}}\right)-\Phi\left(\frac{\mu_{i-1}-X_{t}^{\prime} \beta}{\sigma_{t}}\right) & \text { if } \quad 1<i<M \\
1-\Phi\left(\frac{\mu_{M-1}-X_{t}^{\prime} \beta}{\sigma_{t}}\right) & \text { if } \quad i=M,\end{cases}
\end{aligned}
$$

where $\Phi$ is the standard normal cumulative distribution function. In order to estimate the probabilities $\operatorname{Pr}\left[Z_{t}=s_{i} \mid X_{t}, D_{t}\right]$, we need estimates for the $q \times 1$ vector $\beta$, the $M-1$ threshold parameters, and the parameter $\gamma_{1}$ of the conditional heteroscedasticity specification.

In order to interpret the signs of the coefficients and their impact on observing a given category $s_{i}$, the following partial derivatives apply:

$$
\frac{\partial P\left(Z_{t}=s_{i} \mid X_{t}, D_{t}\right)}{\partial X_{u t}}= \begin{cases}-\phi\left(\frac{\mu_{1}-X_{t}^{\prime} \beta}{\sigma_{t}}\right) \frac{\beta_{u}}{\sigma_{t}}, & i=1 \\ {\left[\phi\left(\frac{\mu_{i-1}-X_{t}^{\prime} \beta}{\sigma_{t}}\right)-\phi\left(\frac{\mu_{i}-X_{t}^{\prime} \beta}{\sigma_{t}}\right)\right] \frac{\beta_{u}}{\sigma_{t}},} & 1<i<M \\ \phi\left(\frac{\mu_{M-1}-X_{t}^{\prime} \beta}{\sigma_{t}}\right) \frac{\beta_{u}}{\sigma_{t}}, & i=M .\end{cases}
$$

\section{A.2 Estimation of the Model}

The model is estimated with maximum likelihood, assuming a normally distributed error term. In order to write the likelihood function in a compact form we introduce some additional notation, following McKelvey and Zavoina (1975) who derive results for the homoscedastic case. Consequently, we will extend their results to the heteroscedastic case. Let

$$
\Phi_{t, k}=\Phi\left(\frac{\mu_{k}-\sum_{i=1}^{q} \beta_{i} X_{i t}}{\sigma_{t}}\right), \quad k=1, \ldots, M
$$

and

$$
\phi_{t, k}=\phi\left(\frac{\mu_{k}-\sum_{i=1}^{q} \beta_{i} X_{i t}}{\sigma_{t}}\right), \quad k=1, \ldots, M
$$


Define $Y_{t, k}$ is an indicator which takes the value of 1 if $Z_{t}=s_{k}$, and 0 otherwise. The log-likelihood for one observation is given by

$$
L_{t}=\sum_{k=1}^{M} Y_{t, k} \log \left(\Phi_{t, k}-\Phi_{t, k-1}\right) .
$$

Let $\delta_{i, j}$ be the Kronecker delta defined by:

$$
\delta_{i, j}= \begin{cases}1, & \text { if } i=j \\ 0, & \text { otherwise. }\end{cases}
$$

The partial derivatives are given by ${ }^{16}$

$$
\begin{gathered}
\frac{\partial L_{t}}{\partial \mu_{u}}=\sum_{k=1}^{M} Y_{t, k}\left[\frac{\phi_{t, k} \delta_{k, u}-\phi_{t, k-1} \delta_{k-1, u}}{\Phi_{j, k}-\Phi_{j, k-1}}\right] \frac{1}{\sigma_{t}}, \quad u=1, \ldots, M-1 \\
\frac{\partial L_{t}}{\partial \beta_{u}}=\sum_{k=1}^{M} Y_{t, k}\left[\frac{\phi_{t, k-1}-\phi_{t, k}}{\Phi_{t, k}-\Phi_{t, k-1}}\right] \frac{X_{u t}}{\sigma_{t}}, \quad u=1, \ldots, q \\
\frac{\partial L_{t}}{\partial \gamma_{1}}=\sum_{k=1}^{M} Y_{t, k}\left[\frac{\phi_{t, k-1}\left(\mu_{k-1}+\sum_{i=1}^{q} \beta_{i} X_{i t}\right)-\phi_{t, k}\left(\mu_{k}+\sum_{i=1}^{q} \beta_{i} X_{i t}\right)}{\Phi_{t, k}-\Phi_{t, k-1}}\right] \frac{\gamma D_{t}}{\sigma_{t}^{3}} .
\end{gathered}
$$

The function in Expression (3) is maximized numerically, using the analytical gradient vector given above. The covariance matrix for the parameters is estimated by quasi-maximum likelihood, which is heteroscedasticity-consistent.

\footnotetext{
${ }^{16}$ c.f. McKelvey and Zavoina (1975)
} 


\section{References}

Beber, A., and C. Caglio (2003): "Order Submission Strategies and Information: Empirical Evidence form the NYSE," Discussion Paper, University of Lausanne, NEWFIN Univesità Bocconi.

Bikhchandani, S., and S. Sharma (2000): "Herd Behavior in Financial Markets: A Review," IMF Working Paper WP/00/48.

Bisière, C., and T. Kamionka (2000): "Timing of Orders, Order Aggressiveness and the Order Book at the Paris Bourse," Annales d'Economie et de Statistique, 60, 43-72.

Chan, L. K. C., N. Jegadeesh, and J. Lakonishok (1996): "Momentum Strategies," Journal of Finance, 51(5), 1681-1713.

Easley, D., N. M. Kiefer, M. O'Hara, and J. B. Paperman (1996): "Liquidity, Information, and Infrequently Traded Stocks," Journal of Finance, 51(4), 1405-1437.

Greene, W. H. (1997): Econometric Analysis. Prentice Hall, Upper Saddle River, New Jersey.

Hall, A. D., and N. Hautsch (2004): "A Continuous-Time Measurement of the BuySell Pressure in a Limint Order Book Market," Discussion Paper, Institute of Economics, University of Kopenhagen.

HARRIs, L. (2003): Trading and Exchanges. Market Microstructure for Practitioners. Oxford University Press.

Harris, L., And J. Hasbrouck (1996): "Market vs. Limit Orders: The SuperDOT Evidence on Order Submission Strategy," Journal of Financial and Quantitative Analysis, 31(2), 213231.

Hasbrouck, J. (1991): "Measuring the Information Content of Stock Trades," Journal of Finance, 46, 179-207.

Hausman, J. A., A. W. Lo, and A. C. MacKinlay (1992): "An Ordered Probit Analysis of Transaction Stock Prices," Journal of Fiancial Economics, 31, 319-379.

Heflin, F., And K. W. Shaw (2004): "Trade Size and Informed Trading: Which Trades Are "Big"?," Journal of Financial Research, forthcoming.

Kavajecz, K. A., and E. R. Odders-White (2001): "An Examination of Changes in Specialists' Posted Price Schedules," Review of Financial Studies, 14, 681-704.

Lee, C. M. C., B. Mucklow, and M. J. Ready (1993): "Spreads, Depths, and the Impact of Earnings Information: An Intraday Analysis," Review of Fiancial Studies, 6, 345-374.

Lee, C. M. C., and M. J. Ready (1991): "Inferring Trade Direction from Intraday Data," Journal of Finance, 46(2), 733-746.

McKelvey, R. D., And W. Zavoina (1975): "A Statistical Model for the Analysis of Ordinal Level Dependent Variables," Journal of Mathematical Sociology, 4, 103-120. 
Petersen, M. A., And S. Umlauf (1994): "Quote Revisions and the Quoted Size: an Empirical Analysis," Working Paper, Kellogg School of Management.

Pohlmeier, W., And F. Gerhard (2000): "Identifying Intraday Volatility," Working Paper, Center of Finance and Econometrics, University of Konstanz. 